\title{
ERB Oncogene Family
}

National Cancer Institute

\section{Source}

National Cancer Institute. ERB Oncogene Family. NCI Thesaurus. Code C18507.

Retrovirus-associated DNA sequences originally isolated from, or related to, the avian erythroblastosis virus (AEV). The c-erbA proto-oncogene encodes the thyroid hormone receptors, and the c-erbB family codes for the epidermal growth factor receptor (erbB-1, EGFR) and related receptors (erbB-2, erbB-3). 